\title{
Forme et réforme organisationnelles des Chambres d'agriculture. Une lecture à partir des régimes d'action des conseillers
}

Form and reform in the organization of the Chambers of agriculture: a analysis from advisors' activities

Claude Compagnone, Bruno Lemery, Sandrine Petit, François Kockmann et Pascale Moretty

\section{(2) OpenEdition}

\section{Journals}

Édition électronique

URL : http://journals.openedition.org/economierurale/4095

DOI : $10.4000 /$ economierurale.4095

ISSN : 2105-2581

Éditeur

Société Française d'Économie Rurale (SFER)

Édition imprimée

Date de publication : 15 octobre 2013

Pagination : 41-58

ISSN : 0013-0559

Référence électronique

Claude Compagnone, Bruno Lemery, Sandrine Petit, François Kockmann et Pascale Moretty, « Forme et réforme organisationnelles des Chambres d'agriculture. Une lecture à partir des régimes d'action des conseillers », Économie rurale [En ligne], 337 | septembre-octobre 2013, mis en ligne le 15 octobre 2015, consulté le 19 avril 2019. URL : http://journals.openedition.org/economierurale/4095 ; DOI : 10.4000/economierurale.4095 


\title{
Forme et réforme organisationnelles des Chambres d'agriculture Une lecture à partir des régimes d'action des conseillers
}

\author{
Claude COMPAGNONE • AgroSup Dijon, UMR Cesaer, Dijon \\ Bruno LEMERY • AgroSup Dijon, UMR Cesaer, Dijon \\ Sandrine PETIT • Inra, UMR Cesaer, Dijon \\ François KOCKMANN • Chambre d'agriculture de Saône-et-Loire, Macon \\ Pascale MORETTY • Chambre d'agriculture de Saône-et-Loire, Macon
}

Le travail réalisé à partir de l'analyse des discours des agents des Chambres d'agriculture de la Saône-et-Loire et de la Haute-Savoie sur leurs activités et sur le fonctionnement de leur organisation, a permis de définir deux axes d'analyse. Le premier s'appuie sur une tension entre un régime d'action du proche et un régime du distant, et le second sur une tension entre la mise en œuvre d'un service marchand et d'un service public. À partir du croisement de ces deux axes, une grille d'analyse, qui différencie quatre espaces d'activités, a été construite. Les différents services de ces deux Chambres ont été positionnés sur cette grille. Ont ainsi émergé des profils contrastés de ces deux Chambres. Ces profils ont été mis en perspective avec l'autonomie ou l'hétéronomie de ces structures.

MOTS-CLÉS : organisation, régime d'action, chambre d'agriculture, sociologie, activités de conseil

Form and reform in the organization of the Chambers of agriculture: a analysis from advisors' activities

The article is drawn up from an analysis of the discourses that advisors of the Chambers of agriculture of Saône-et-Loire and Haute-Savoie develop about their activities and functioning of their company. On this basis, we define two axes of analysis. The first axe is grounded in a tension between the action regime of the "close" and the action regime of the "distant"; the second axe represents a tension between the implementation of a public service and a commercial service. Crossing the two axes provides a key for understanding advisory activities with four areas of action. This key was used to qualify the different departments which are made of the two Chambers. Contrasted profiles of action emerged out for each Chamber. These profiles were connected with the "autonomy" or the "hetoronomy" of these agricultural bodies.(JEL:Q16)

KEYWORDS: organization, action regime, Chambers of agriculture, sociology, advisory activities

Faire émerger des profils d'activités des Chambres

1. Une reconfiguration du champ du conseil

Si les Chambres d'agriculture départementales ont historiquement joué, en France, un rôle central dans la modernisation de l'agriculture (Rémy et al., 2006), elles se trouvent actuellement profondément affectées par des changements qui les obligent à redéfinir et réagencer leurs activités (Lémery, 2006 ; Compagnone et Lémery, 2009 ; Albaladejo et al., 2009 ; Faure et Compagnone, 2011 ; Faure et al., 2011). 
Elles doivent non seulement prendre en compte dans leur travail de nouvelles exigences sociétales, principalement en matière sanitaire et environnementale (Brives, 2006), mais aussi composer, dans l'orientation des pratiques agricoles, avec de nouveaux acteurs - associations, collectivités territoriales, agences, etc. - (Ruault et Lémery, 2009 ; Auricoste et al., 2011). Elles sont, de plus, confrontées à une diminution des ressources économiques sur lesquelles elles s'appuyaient jusqu'à présent. De moins en moins bien dotées en différentes subventions nationales ou locales, elles comptent principalement aujourd'hui sur les ressources tirées de l'impôt sur le foncier non bâti - dont le taux varie d'un département à l'autre et sur la vente de prestations de conseil (Mundler, 2006 ; Petit et al., 2011). Dans ce contexte, elles sont tout d'abord amenées à déployer leurs activités de manière contractuelle avec d'autres acteurs, à développer une gamme de services payants et à entrer en concurrence avec d'autres organisations telles que les organismes économiques de l'agrofourniture, les cabinets de consultants, voire les autres Organisations professionnelles agricoles (OPA). Cette concurrence s'exerce aussi bien en matière de conseil aux agriculteurs que d'offre de services aux collectivités territoriales. Elles sont ensuite contraintes de développer de nouvelles formes de coopération entre elles. La Réforme générale des politiques publiques les oblige ainsi à mutualiser à l'échelon régional une partie de leurs activités afin de pouvoir réaliser des économies dans leur mode de fonctionnement.

La marchandisation des prestations de conseil devient dans ce cadre un enjeu majeur de la survie et du positionnement mutuel de ces organisations (Petit et al., 2011). En effet, les organismes économiques de l'agrofourniture (coopératives et entreprises privées) tendent de plus en plus à commercialiser en tant que tel le conseil aux agriculteurs. Ce conseil devient alors une activité à part entière qui n'est plus intégrée comme antérieurement à la vente d'un produit, qu'il s'agisse, par exemple, de pesticides, d'aliments pour bétail ou de matériels agricoles. On observe ainsi dans le domaine viticole, depuis le milieu des années 2000, le développement - principalement par des entreprises privées - de la vente de prestations pour le suivi sanitaire des vignes ou l'information et la formation des viticulteurs sur les nouvelles exigences réglementaires (Compagnone et Golé, 2011). De leur côté, les Chambres sont conduites à différencier des offres de prestations marchandes de celles totalement ou partiellement financées par les ressources tirées de l'impôt sur le foncier non bâti. L'enjeu est pour elles, non seulement de pouvoir maintenir leurs activités en dégageant des moyens économiques suffisants, mais aussi d'être capables de prendre pied dans un espace du conseil qui se structure de plus en plus sous la forme d'un marché. Pour le dire rapidement, pour pouvoir être présent dans cet espace et continuer à " entrer chez les agriculteurs », il leur faut être en mesure de vendre leurs services de la même manière que peuvent le faire leurs concurrents.

On assiste donc à une reconfiguration du champ ${ }^{1}$ du conseil. Cette reconfiguration porte tout aussi bien sur la nature des forces qui orientent ce champ, et donc sur la nature des ressources que chacun doit posséder pour s'y inscrire au mieux par rapport aux autres, que sur les types d'acteur qui y prennent position. La légitimité

1. Le concept de champ ici utilisé est à entendre au sens que lui donne Bourdieu en 1992. Il désigne « un réseau ou une configuration de relations objectives entre des positions », positions renvoyant elles-mêmes à une certaine « situation... dans la structure de la distribution des différentes espèces de pouvoir dont la possession commande l'accès aux profits spécifiques qui sont en jeu dans le champ » (Bourdieu et Wacquant, 1992). 
de l'activité de conseil ne tient plus, ou du moins plus seulement, au mandat et à la licence (Hughes, 1996) que le conseiller agricole peut recevoir de la part des représentants de la profession agricole pour pouvoir l'exercer. D'autres opérateurs interviennent avec d'autres conceptions ou d'autres visées sur l'agriculture (Lémery, 2006 ; Compagnone et Lémery, 2009). Le modèle de l'exploitation familiale, voire même de l'agriculteur professionnel menant de front l'ensemble des tâches de production propres à son système, est contesté. Des formes de délégation de la conduite de ces tâches apparaissent, qu'il s'agisse de faire assumer par d'autres acteurs la réalisation de travaux agricoles ou de diagnostics pour la mise en œuvre de ces travaux, ou encore de leur faire définir les orientations mêmes de la production. Les organismes économiques, dont les agents sont fortement présents sur les territoires et dont les activités s'inscrivent déjà dans le cadre de relations marchandes, possèdent des ressources adaptées pour prendre position dans ce nouveau champ (Hellec, 2009; Compagnone et Golé, 2011 ; Goulet, 2011).

\section{Caractéristiques des activités et profils des Chambres}

Dans ce contexte, comment se positionnent, les unes par rapport aux autres, les activités que les Chambres d'agriculture mènent à destination des agriculteurs ? En nous posant cette question, nous souhaitons nous donner les moyens de caractériser les profils organisationnels des Chambres en fonction des traits à partir desquels les différents agents de ces structures définissent et qualifient euxmêmes leurs activités. Le travail de définition qu'ils opèrent prend corps, en situation d'instabilité, dans le cadre d'un débat, auquel ils participent plus ou moins, sur ce que doit être l'activité des conseillers des Chambres. Dans leur discours, ils opposent alors les activités à partir d'un certain nombre de traits saillants et étayent leurs propos par des arguments socialement acceptables en circulation dans l'espace social du conseil en agriculture, sur lesquels ils prennent appui non seulement pour expliquer ce qu'ils font ou doivent faire, mais aussi pour le justifier. L'identification de ces arguments, et leur condensation dans des critères synthétiques, nous permettront de construire, dans une première partie de cet article, une grille d'analyse utilisable pour établir des profils différenciés de Chambres. Nous nous appuierons dans cette démarche principalement sur des outils issus de la sociologie pragmatique (Boltanski et Thévenot, 1991 ; Thévenot, 2006).

Toutefois, une telle approche, focalisée sur les aspects qui font socialement sens dans un contexte de transformation donné, nous renseigne assez peu sur les interactions professionnelles concrètes dans lesquelles sont impliqués les agents dans leur activité. Ces interactions, à la fois, contribuent aux positions argumentatives des uns et des autres sur ce que doivent être les activités des conseillers, et sont pour partie influencées par elles. Nous relèverons donc, dans une seconde partie, la diversité de ces interactions pour situer celles où se jouent des manières différentes d'être conseiller. Nous nous interrogerons de plus, dans le cadre d'une réflexion plus politique, sur le lien entre la forme des profils des Chambres et la façon dont le pouvoir se trouve, dans ces Chambres, exercé par différents acteurs du champ du développement. Nous nous situerons alors dans une perspective interactionniste, perspective qui s'intéresse à la nature des interactions entre individus et à l'ordre social qui en découle (Strauss, 1992).

Pour mener à bien cette tâche, nous nous appuierons principalement sur les résultats d'une enquête que nous avons menée en 2008 et 2009 dans les Chambres d'agriculture, de Saône-et-Loire et de 
Haute-Savoie ${ }^{2}$, auprès d'élus du bureau du conseil d'administration, des membres de la direction ${ }^{3}$ et de différents agents des services. Cette enquête a donné lieu à la réalisation d'une vingtaine d'entretiens compréhensifs semi-directifs dans chaque structure. Ces entretiens avaient pour visée de faire décrire aux personnes rencontrées : leur trajectoire personnelle ; les types d'activités qu'elles mènent et la manière dont elles les agencent; les modes de coordination développés avec d'autres agents de la structure ou d'autres organisations agricoles ; la manière dont leur travail est orienté, encadré et contrôlé ; les ressources, cognitives, sociales et matérielles, sur lesquelles elles s'appuient ; leur perception du fonctionnement de leur service et de la structure dans son ensemble. Nous utiliserons en complément des résultats tirés d'un travail effectué en 2012 sur les sept Chambres départementales d'agriculture de la Région Rhône-Alpes. Ce travail a été conduit auprès d'une quinzaine d'agents de chacune de ces Chambres en suivant le même mode d'investigation que pour celles de Saône-et-Loire et de HauteSavoie.

\section{Définition des types d'activités de conseil}

Dans les entretiens, lorsque les différents agents parlent de leurs activités auprès des agriculteurs en tant que producteurs agricoles, ils s'appuient sur une série de critères agencés plus ou moins explicitement par couples d'oppositions. Ces couples d'oppositions leur permettent de définir ce qu'ils font ou ce qu'ils voudraient ou devraient faire par rapport à d'autres acteurs

2. La Chambre de Haute-Savoie a depuis 2012 fusionné avec celle de Savoie pour ne plus former qu'une seule et même entité.

3. C'est-à-dire du directeur et des chefs de service ou responsables de pôles. du conseil. Ces couples, pour ne citer que les principaux, sont :

- conseiller de secteur généraliste ou animateur de groupement agricole ${ }^{4}$ basé sur le terrain $v s$ conseiller spécialiste basé au siège de la Chambre ;

- organisation des activités par projet $v s$ organisation des activités par missions pérennes ;

- appui technique aux agriculteurs sur leurs exploitations vs appui technique aux responsables professionnels pour leur activité politique ;

- appui technique aux agriculteurs $v s$ appui aux projets de développement territorial ;

- prestations aux agriculteurs financées par l'impôt $v s$ prestations payantes financées directement par les bénéficiaires ;

- actions de développement vs actions marchandes.

Ces couples d'oppositions renvoient à des formes différentes de coordination entre acteurs. On peut identifier, dans la façon dont ces oppositions se trouvent articulées entre elles, deux dimensions. La première repose sur l'opposition proche/distant, et la seconde sur l'opposition public/marchand.

\section{Des régimes d'action du proche et du distant}

La première dimension correspond à la tension entre deux régimes d'action différents dans lesquels les agents se trouvent engagés dans leur travail avec les agriculteurs : celui du proche et celui du distant. À chacun de ces régimes sont associés, comme nous allons le voir, des formes de coordination entre acteurs, des façons de nouer et d'entretenir les liens sociaux,

4. Les Groupements de développement agricole (GDA ou GVA) sont présents en Haute-Savoie tandis qu'ils ont disparu au début des années 1980 en Saône-et-Loire. Nous parlerons dans la suite du texte indifféremment de conseillers d'entreprise ou de conseillers de secteur. 
des compétences, des manières de juger et d'apprécier les choses, des positionnements géographiques et sociaux spécifiques. Ces régimes sont des formes pures que les conseillers peuvent plus ou moins combiner dans leur activité ordinaire auprès d'agriculteurs différents mais plus difficilement auprès d'un même agriculteur.

Ces deux régimes tiennent en partie $\mathrm{du}$ régime d'engagement familier et $\mathrm{du}$ régime d'engagement en plan décrit par Laurent Thévenot (2006), sans pour autant s'y superposer complètement. Le régime d'engagement familier «se concrétise par l' "aise" que l'on ressent dans un environnement connu où tout est à disposition et où la familiarisation fait que l'on s'y retrouve "les yeux fermés", par une sorte de pleine disponibilité et de pleine convergence entre le corps, les êtres et les objets » alors que «le modèle privilégié par le plan est celui de l'action caractéristique des logiques du contrat et du projet propres au monde des relations organisées »(Genard, 2011). Si le régime de l'engagement en plan colle assez bien à notre régime du distant, celui en familiarité, en mettant l'accent sur la coordination entre soi et l'environnement avant celle avec autrui, et en réduisant cette dernière « à un genre d'intimité routinière » (Thévenot, 2006), est bien trop restrictif. Dans ce sens, notre régime d'action du proche, intégrera un type de relation sociale qui tiendra plus de la socialité primaire, mis en évidence par Alain Caillé (2007) dans sa théorisation du don, que de cette « intimité routinière » de Thévenot. Caillé distingue en effet socialité primaire et socialité secondaire. La socialité primaire est un type de rapport « dans lequel la personnalité des personnes importe plus que les fonctions qu'elles accomplissent (ce qui n'empêche pas ces fonctions d'exister et d'importer) » alors que dans la socialité secondaire, «les fonctions accomplies par les personnes importent plus que leur personnalité ». La loi du don structure la socialité primaire dans le cadre de réseaux interpersonnels (famille, voisinage, alliance, amitié...) alors que l'impersonnalité prime dans la socialité secondaire (marché, droit, science...). La socialité primaire caractérise bien le type de relation sociale engagée dans notre régime du proche et la socialité secondaire la relation identifiée dans notre régime du distant. Bien que nous précisions le type de socialité propre à ces régimes, nous gardons la notion de régime d'action qui nous semble pertinente pour appréhender l'action «à travers l'équipement mental et gestuel des personnes, dans la dynamique d'ajustement des personnes entre elles et avec des choses » (Corcuff, 1998).

Le régime $d u$ proche, dans le sens où nous l'entendons ici, se caractérise par un mode de coordination et de définition de l'action entre le conseiller et l'agriculteur qui est celui de l'arrangement. C'est en situation et dans l'interaction concrète avec les agriculteurs que se définit le genre de travail à accomplir. Les choses ne sont pas planifiées et prédéfinies a priori. L'arrangement qui s'opère avec l'agriculteur pour définir ce qu'il faut faire, amène le conseiller à articuler des connaissances et des outils divers qui ne sont pas déjà là. Il déploie une compétence particulière pour la détermination de ce qui pose problème et pour l'assemblage d'éléments hétéroclites de réponse. La figure type de ce régime d'action est le conseiller de secteur expérimenté, installé depuis de nombreuses années sur une même zone et qui possède une longue expérience de travail avec les agriculteurs d'un même groupe de développement agricole. Pour que ce travail puisse se faire, il faut une proximité géographique, car pour construire l'action, il faut être là au bon moment avec les agriculteurs. La présence de l'agent de Chambre ne peut pas être intermittente mais doit être constante. Cette référence au proche apparaît dans les entretiens dans la forte valorisation « du terrain » 
ou « du secteur ». Être sur le terrain, c'est, d'une certaine façon, « l'habiter » (De Certeau, 1990), c'est-à-dire arpenter les lieux et rencontrer les gens. Dans ce sens, la proximité est aussi sociale. Il semble clair pour les différents interviewés que la légitimité de l'action de leur Chambre repose sur leur inscription sur le terrain. S'il y a débat entre les services qui portent ce type d'approche et ceux qui se trouvent plutôt dans un régime du distant, c'est sur le fait de savoir si la légitimité de l'action de la Chambre repose entièrement ou seulement en partie sur ce type d'engagement dans l'action.

Le régime $d u$ distant, quant à lui, se caractérise par un mode de coordination entre acteurs qui est du domaine de la convention. L'engagement dans l'action des agents, la durée de cette action et les moyens mis en œuvre, sont définis de manière conventionnelle, voire contractuelle. Les actions engagées sont donc prédéfinies et planifiées. Elles s'intègrent dans le cadre de projets à la durée de vie déterminée, où les rôles des uns et des autres sont spécifiés. La figure type de ce régime est le conseiller spécialisé qui n'intervient sur l'exploitation que sur une question précise. Il peut s'agir, par exemple, de régler un problème de taux de cellules trop élevé dans le lait des vaches, ou encore, de réaliser un plan de fumure chez un agriculteur. Dans la relation des agents avec les agriculteurs, une distance géographique est entretenue, puisque l'intervention du conseiller se fait de manière ciblée et ponctuelle, à la demande des agriculteurs, principalement à partir du siège de la Chambre d'agriculture. Cette intervention est centrée sur un sujet précis et elle est réalisée par un spécialiste. Cette distance géographique se trouve redoublée par une distance sociale, dans le sens où les agriculteurs ne sont rencontrés que ponctuellement.

\section{Services marchands et services publics}

La deuxième dimension sur laquelle nous nous appuyons dans notre analyse renvoie, quant à elle, à l'univers social, aux « mondes » pour reprendre les termes de Boltanski et Thévenot (1991), auxquels se réfèrent les agents pour expliquer, défendre ou justifier ce qu'est ou ce que devrait être la visée d'intervention de leur organisation ou, à défaut, la leur au sein de cette organisation. Cette dimension est celle de la nature publique ou marchande du service apporté aux agriculteurs. Elle réfère l'action des agents à un cadre plus large que celui défini par l'ensemble des interactions entretenues avec d'autres acteurs dans et hors l'organisation. Ce cadre est celui du corps social dans son ensemble qui, bien qu'absent concrètement dans l'interaction, met à la disposition des agents un répertoire de visées socialement acceptables et de registres de justification sur lesquels ils peuvent prendre appui pour dire ce qu'ils font. On se situe dans le régime de l'action justifiable (Boltanski et Thévenot, 1991 ; Thévenot, 2006). Dans ce sens, cette deuxième dimension est de nature plus idéologique et fournit aux agents un « horizon de sens » à leur pratique.

Comme nous avons pu nous en rendre compte dans la description précédente, un lien existe entre ces régimes d'action et le type de rapport économique dans lequel les agents se trouvent engagés dans leurs interactions avec les agriculteurs. Les questions autour de l'orientation marchande ou publique des services délivrés par la Chambre traversent les propos de toutes les personnes rencontrées. Les débats ne tournent pas autour du fait de développer ou non des services payants, la facturation des conseils étant, ces dernières années, devenue banale. Ils se concentrent sur la visée marchande ou non des prestations déployées et sur le sens du métier de conseiller qui en découle (Petit et al., 2011).

$\mathrm{Si}$, dans les entretiens, la distinction visée marchande $v s$ visée non marchande est bien présente, vient s'y ajouter toute une série de traits qui complexifient la 
caractérisation de ces deux formes d'action. Dans les discours des agents, une action de service public non seulement n'a pas de visée lucrative mais elle se trouve, de plus, financée de manière plus ou moins importante par l'impôt. Elle est donc proposée gratuitement ou à un prix inférieur au prix coûtant, ce prix étant identique pour l'ensemble des agriculteurs, quelles que soient les ressources investies dans la réalisation de l'action. De ce fait, les actions de ce type ne prennent pas toujours, à proprement parler, la forme de prestations, dans le sens où elles ne sont pas standards et où elles s'adaptent au cas par cas, sans avoir forcément été définies à l'avance. À l'opposé, un service marchand correspond à la mise en œuvre de prestations payantes, avec une visée lucrative. Le prix des prestations est individualisé et adapté à l'importance de l'investissement nécessaire à leur réalisation. Ces prestations correspondent, dans le cadre d'un échange contractualisé, à une diversité de produits prédéfinis, commercialisables, c'est-à-dire ayant une valeur économique. Les bénéficiaires de ces prestations, les « clients », ne sont alors pas uniquement les agriculteurs, mais peuvent être des collectivités, des associations, des administrations, etc.

Chacun des deux éléments de cet axe service public/service marchand se superpose plus ou moins bien à l'un ou l'autre régime d'action décrit précédemment. Ainsi, la proximité de terrain des agents avec les agriculteurs renvoie à une forme de relation qui ne cadre pas avec une relation marchande comme celle que les Chambres, de plus en plus soucieuses de leur équilibre financier, entendent promouvoir au travers de prestations payantes. L'objectif est plutôt de pouvoir fournir un conseil pour partie gratuit ou qui s'inscrit dans une logique de service public. Dans ce modèle d'action, c'est une socialité primaire avec les agriculteurs qui est défendue, pour permettre à des relations de confiance de s'instaurer et à des arrangements de s'opérer dans la manière de traiter les problèmes. Le principe d'une commercialisation du conseil, passant par une prestation préétablie, est vu comme empêchant ces arrangements alors que, poussés de plus en plus à faire payer leurs interventions, les conseillers sont contraints de facturer leur service. Les conseillers d'entreprise ou les animateurs de groupements prennent assez généralement position contre cette tendance. Leurs actions sont en partie financées par l'impôt, c'est-à-dire par les fonds propres de la Chambre, mais peuvent aussi l'être par des subventions de collectivités - dans le cadre d'animation territoriale, par exemple -, ou par des prestations vendues aux collectivités. À l'opposé, les agents dans le régime du distant vont plus facilement valoriser le développement de services marchands. Les actions à conduire auprès des agriculteurs étant ponctuelles et, en principe, parfaitement définies, elles ont un coût évaluable et peuvent, en conséquence, être facturées. L'instauration de ce type de relation doit permettre, selon eux, de gagner en précision et en stabilité dans la définition de leurs tâches, et de voir la valeur de leur intervention reconnue. L'enjeu est de pouvoir entrer en concurrence avec les services des entreprises de l'agrofourniture ou d'autres organisations professionnelles agricoles (coopératives, syndicats de contrôle laitier, etc.) très présentes sur le terrain et pratiquant, elles, de manière ordinaire, la vente de prestations.

Toutefois, si les deux éléments de cet axe service public/service marchand s'inscrivent plus favorablement dans l'un ou l'autre régime d'action, ils ne s'y confondent pas pour autant, des activités de services publics pouvant être, par exemple, développées dans le régime du distant. Une telle situation nous conduit à organiser notre analyse en croisant ces deux axes afin d'observer les espaces d'activités qui peuvent se révéler et voir la 
façon dont les services des Chambres s'y positionnent.

\section{Une grille d'analyse et son usage}

Quatre espaces d'activités apparaissent : le public distant, le public proche, le marchand distant, le marchand proche (I, II, III et IV, dans le schéma 1). Notons que Thévenot (2006) n'opère pas, quant à lui, le type de croisement que nous effectuons ici. S'il distingue un régime du familier, un régime du plan et un régime de la justification, cette distinction se base principalement sur l'étendue de l'espace social - du plus étroit au plus large - impliqué dans l'action et le contrôle de l'action avec les objets et les personnes. Si le croisement que nous effectuons nous semble possible, c'est parce que nous nous intéressons à la façon dont, selon le registre de justification sur lequel s'appuient les acteurs pour dire ce que doit être l'action de leur organisation, prennent corps des formes d'engagement dans l'action auprès des agriculteurs spécifiques qui tiennent du régime du proche ou du distant.

Ces quatre espaces sont caractérisés en fonction de la nature du conseil délivré, des modalités de sa mise en œuvre et de son mode de financement. La grille représentée ci-dessous (schéma 1) permet de rendre compte de la façon dont ces différents critères se trouvent renseignés dans chacun des quatre espaces considérés. Elle a une valeur synthétique dans la mesure où elle permet de condenser, à partir des deux axes précédemment décrits, les principaux critères de distinction entre ces quatre espaces d'activités.

Bien que cette grille ait été construite à partir de traits qui apparaissent en tension

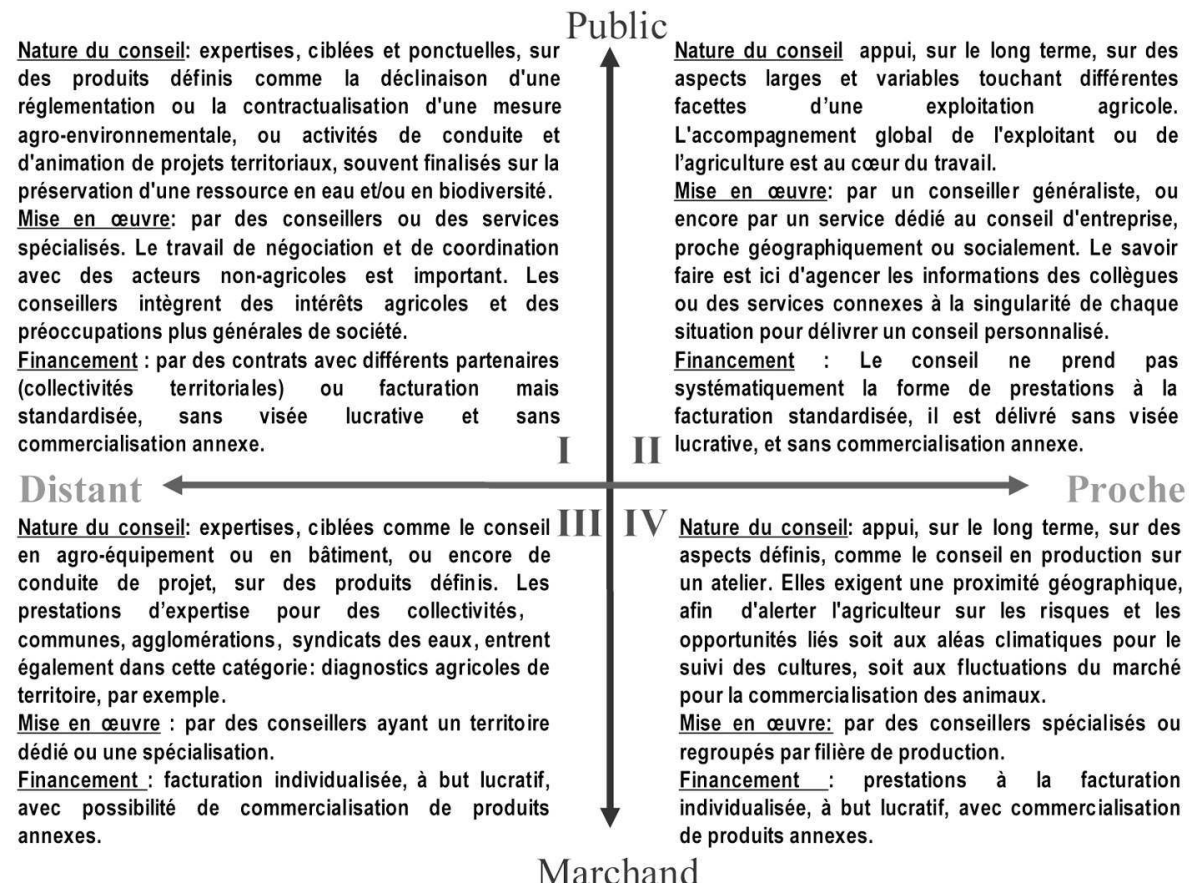


dans le discours des agents sur ce que doit être ou ne pas être leur activité de conseil, elle peut être utilisée pour positionner, en fonction de la description qu'en font ces agents, les activités concrètes des conseillers et des services de la Chambre.

Il va de soi que, selon la nature de ces activités, ces espaces sont plus ou moins occupés. Mais de manière générale, l'enquête nous montre que les activités des conseillers se trouvent de manière dominante dans l'un ou l'autre. Tenir toutes les positions en même temps est impossible, même si un panachage entre activités peut s'opérer à la marge. Ainsi, le fait d'être conseiller sur un secteur (dans un régime du proche) n'empêche pas d'intervenir à l'échelle du département pour la formation des agriculteurs (régime du distant). Pour donner des exemples, les conseillers qui s'investissent principalement dans la formation des agriculteurs sur des aspects réglementaires se trouvent dans l'espace I. Ceux implantés sur des territoires et qui animent des groupes techniques (Saôneet-Loire) ou de développement territoriaux (Haute-Savoie), se situent dans l'espace II. Les conseillers spécialisés, comme les conseillers en bâtiments ou en grandes cultures, sont positionnés dans l'espace III. L'espace IV qui correspond assez bien dans sa définition au travail réalisé par les technico-commerciaux des organismes économiques, peut aussi être investi. En HauteSavoie, le travail des agents du service « Traite et qualité du lait » s'inscrit parfaitement dans ce cadre.

Nous pouvons étendre l'usage de la grille à la représentation d'un service vu alors comme la somme des activités de ses agents. Les services étant des unités complexes aux activités parfois hétérogènes, ils apparaissent alors, comme nous allons le voir, sous la forme de «patatoïdes » pouvant recouvrir plusieurs espaces d'activités. Cette représentation graphique montre des profils organisationnels différents des Chambres d'agriculture.

Pour montrer la logique suivie dans ce positionnement, nous avons tout d'abord représenté dans le schéma 2 les profils d'activités des deux plus gros services de la Chambre d'agriculture de Saôneet-Loire : le service Agronomie, gestion de l'espace, environnement (SAGE) et le service Développement, conseil

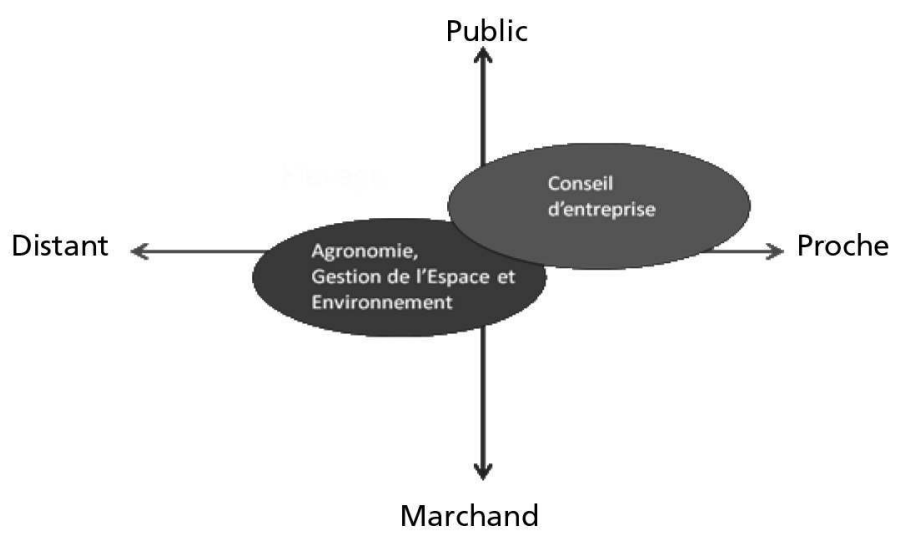

Source : les auteurs. 
d'entreprise, formation, installation-emploi. Ils occupent dans la grille des positions symétriques. Le premier se trouve essentiellement dans l'espace du distant et du marchand, alors que le second se situe dans celui du public et du proche. Mais l'un et l'autre empiètent aussi, par une série d'activités plus secondaires, sur les autres espaces. En s'appuyant sur les codes (I, II, III et IV) attribués à chacun des espaces d'activités, on peut rendre compte de la distribution des activités.

Le service Agronomie, gestion de l'espace, environnement, composé de conseillers spécialisés et de conseillers en développement local, se situe essentiellement dans l'espace du distant et du marchand. Il réalise des prestations auprès des communes (recyclage des boues de station d'épuration), du conservatoire des sites naturels (gestion de la biodiversité) et des syndicats des eaux (diagnostics et animation de programmes d'actions sur les bassins d'alimentation de captages) (III). Il développe, de plus, des outils de conduite des grandes cultures dans le cadre de prestations marchandes (III). Toutefois, il empiète aussi sur l'espace du distant et du public. Il assure ainsi des missions de service public concernant la réglementation agrienvironnementale ou l'urbanisme, conduit des travaux de recherche de références en agronomie et anime des démarches territoriales (I). Il se positionne, enfin, dans l'espace du proche et du marchand quand il effectue de manière pérenne des cycles de formation auprès de groupes locaux d'agriculteurs (IV).

Le service Développement, conseil d'entreprise, formation, installation-emploi, composé de conseillers d'entreprise territorialisés et de conseillers spécialisés, se trouve, quant à lui, principalement dans l'espace du proche et du public. Il assure en priorité, via un réseau de conseillers territorialisés, un conseil de proximité au niveau global de l'exploitation (II), pour partie

\section{Encadré 1. Productions agricoles et types de service des Chambres d'agriculture}

- La Haute-Savoie est une terre d'herbages allant des collines aux alpages d'altitude. L'agriculture est orientée vers la fabrication laitière $(80 \%$ des exploitations professionnelles) avec une gamme de fromages sous signes de qualité, notamment AOC (Reblochon, Beaufort, Abondance, Tomme de Savoie...). La viticulture et l'arboriculture sont aussi présentes. Le fort développement économique et touristique du département implique une défense de la place de l'agriculture dans les territoires, qui est assurée par la présence de groupes de développement agricole. Ils sont reliés au pôle Entreprise Territoire et Aménagement qui a en charge l'aménagement foncier et le conseil d'entreprise, présenté dans le schéma 3 en deux équipes. L'installation est devenue une priorité de la Chambre à la suite de l'absorption de l'ADASEA au sein du service formation-installation-transmission-emploi. Les démarches collectives de qualité des produits sont conduites de concert avec les syndicats de défense des produits.

- La Saône-et-Loire est un grand département agricole de par le nombre d'actifs agricoles et d'exploitations. L'agriculture y est diversifiée. Deux grands systèmes de production sont économiquement dominants : l'élevage allaitant et la viticulture. Mais d'autres productions telles que les grandes cultures et l'élevage laitier, ainsi que les productions ovines, caprines, avicoles et porcines sont très présentes. On trouve aussi du maraîchage et de l'horticulture. Les conseillers de la Chambre spécialisés par production sont répartis dans les services Élevage, Viticulture et Agronomie. Cette organisation par filière croise celle par territoire, les conseillers de secteur, qui relèvent $\mathrm{du}$ service Conseil d'entreprise, formant le réseau de proximité aux agriculteurs. La formation, l'installation, le tourisme, le développement des circuits courts sont des thèmes traités dans des services spécifiques. La compétence d'identification animale est une délégation de service public relevant de l'établissement départemental d'élevage, aujourd'hui assurée par les Chambres. 


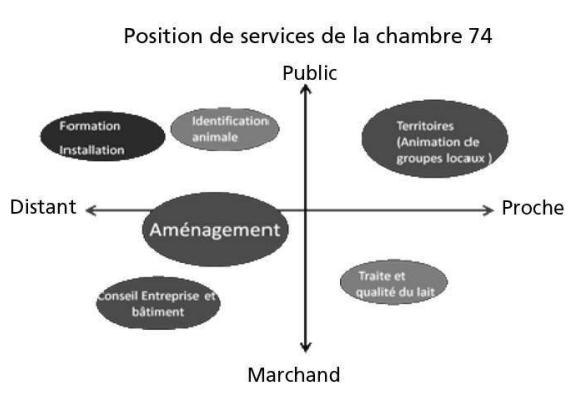

payant. Mais il se positionne aussi dans l'espace du proche et du marchand (IV) car ses conseillers contribuent, en interaction avec les conseillers spécialisés par filière, à l'usage par les agriculteurs d'appuis techniques payants relatifs aux différents ateliers de production. Il mène, de plus, des missions de service public, en particulier en délivrant des informations sur les évolutions de la politique agricole (I).

L'ensemble des principaux services des deux Chambres peut être ainsi positionné sur cette grille en suivant la même logique. Le profil général de chacune d'elles apparaît alors (schéma 3). On constate ainsi, dès le premier regard, que les services de la Chambre de Haute-Savoie sont assez bien répartis, voire éclatés, entre les quatre espaces. Par contre ceux de la Chambre de Saône-et-Loire se trouvent plutôt regroupés à l'intersection des deux axes, sans pour autant se superposer complètement, et occupent très faiblement l'espace du marchand proche. Cet espace est plutôt dévolu jusqu'à présent, dans le département de Saône-et-Loire, aux coopératives d'approvisionnement (Compagnone et al., 2010).

\section{Types d'interactions et gouvernance des Chambres d'agriculture}

Comme nous l'avons indiqué en introduction, une telle approche a la vertu de situer

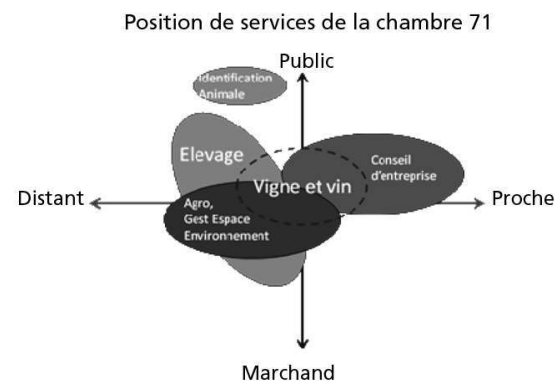

Source : les auteurs.

les activités des agents des Chambres par rapport à des éléments qui font actuellement débat sur les modes d'intervention à déployer auprès des agriculteurs. Toutefois, elle reste muette sur les interactions des acteurs de ces structures. De telles interactions, où se définissent et se jouent les modes d'intervention auprès des agriculteurs, se déroulent entre acteurs de la Chambre mais aussi avec des acteurs extérieurs. Dans cette partie, nous voyons comment les formes d'activités (identifiées précédemment) découlent de ces interactions et sont tenues ensemble. Nous abordons cet aspect en nous centrant tout d'abord, dans une approche synchronique, sur les types d'interactions dans lesquels les agents sont impliqués, et en observant ensuite, dans une approche diachronique, les interactions entre acteurs collectifs qui jouent sur le mode de gouvernance des Chambres étudiées.

\section{Les interactions des agents}

Les interactions dans lesquelles sont impliqués les agents peuvent être différenciées en fonction des scènes ${ }^{5}$ où elles se déroulent (tableau 1). Il y a des scènes d'interactions internes à la Chambre, dans lesquelles les échanges s'opèrent entre les agents ou entre les agents et les élus.

5. Sur l'usage de la métaphore théâtrale dans les sciences sociales, voir Geertz (1999). 
Tableau 1. Les différentes scènes d'interactions des agents

\begin{tabular}{|c|c|c|}
\hline $\begin{array}{l}\text { Liens des agents de la } \\
\text { Chambre }\end{array}$ & $\begin{array}{l}\text { Avec les } \\
\text { agriculteurs }\end{array}$ & $\begin{array}{l}\text { Avec d'autres } \\
\text { acteurs }\end{array}$ \\
\hline $\begin{array}{l}\text { Interne à la } \\
\text { Chambre }\end{array}$ & $\frac{\text { Scène } 1}{\text { Élus de la Chambre }}$ & $\frac{\text { Scène } 2}{\text { Autres agents de la Chambre }}$ \\
\hline $\begin{array}{l}\text { Externe à la } \\
\text { Chambre }\end{array}$ & $\begin{array}{l}\text { Scène } 3 \\
\text { Producteurs agricoles du } \\
\text { département }\end{array}$ & $\frac{\text { Scène } 4}{\text { Partenaires ou clients }}$ \\
\hline
\end{tabular}

Source : les auteurs.

Il y a aussi des scènes externes, où les échanges s'effectuent avec les différents publics (agriculteurs, collectivités, etc.) ou partenaires de la Chambre (financeurs, membres des instances de pilotage ou de concertation, etc.).

Sur deux de ces scènes ( 1 et 3 ), les agriculteurs sont présents, soit au titre de « public » de la structure, soit au titre d'administrateur. Sur la scène des interactions où les agriculteurs interviennent à titre d'administrateurs (scène 1), les agents de la Chambre apportent des informations et références aux responsables professionnels élus pour la réalisation de leur travail d'administrateur mais aussi pour leur activité de représentation et de défense de la profession dans différents lieux sociaux. De leur côté, les élus leur font connaître l'orientation qu'ils souhaitent donner aux activités de la Chambre et contrôlent leur réalisation. Cette interaction s'opère essentiellement entre les membres du comité de direction (directeurs et chefs de services) et ceux du bureau du conseil d'administration de la Chambre.

Mais ce qu'il nous intéresse de voir plus particulièrement ici, c'est ce qui se passe sur les deux scènes ( 2 et 4 ) où les agriculteurs en tant que «public » ne sont plus qu'en arrière-plan de l'action. Sur l'une de ces scènes, les agents ainsi que les élus de la Chambre interagissent avec une grande diversité de partenaires ou de clients (scène 4). Ces acteurs sont des représentants d'OPA (syndicats, instituts techniques, contrôle laitier...), d'organismes économiques (coopératives, négoces...) d'associations (environnementalistes, sportives, professionnelles...), d'administrations (préfecture, direction départementale des Territoires, direction régionale de l'Agriculture et de la Forêt...), d'organismes publics (Agences de l'eau, de l'environnement...) ou de collectivités territoriales (communes, communauté de communes, conseil général, conseil régional...). Ces acteurs interviennent auprès de, ou avec la Chambre pour bénéficier de son expertise technique ou pour orienter son action auprès des agriculteurs. Les interactions déployées prennent alors forme dans des cadres variés.

Un premier cadre est de nature politique, lorsque ces acteurs ont fait valoir par des moyens divers (contestation, négociation, subvention...) leur propre point de vue sur ce que doivent être les pratiques agricoles. Un deuxième est réglementaire, quand l'administration relaie auprès de la Chambre de nouvelles normes et procédures pour que cette dernière les mette en œuvre (par exemple, sur l'usage des pesticides) et lui délègue des tâches de service public. Un troisième est marchand, des prestations (diagnostics territoriaux pour les PLU, SCOT...) étant achetées par des collectivités territoriales pour 
conduire leurs propres politiques. Enfin, un dernier cadre est pratique, lorsque la Chambre coordonne son action avec d'autres organismes pour mener à bien sa politique (par exemple, avec la $\mathrm{Mu}-$ tualité sociale agricole sur l'usage des pesticides).

Une telle situation va pousser la Chambre, d'un côté, à développer des actions de service public auprès des agriculteurs (actions déléguées par l'État et qui seront alors financées par l'impôt sur le foncier non bâti), et de l'autre, celui de prestations marchandes qui ne s'adressent pas aux agriculteurs. Mais elle favorise aussi l'émergence d'un mode de fonctionnement par projet imposé par les financeurs publics (État, régions, départements, agences) pour l'attribution de subventions. Ces financeurs ont, avec un faible pourcentage de financement de chaque projet, une réelle capacité d'orientation des actions entreprises par une Chambre (par exemple, pour l'accompagnement des agriculteurs en difficulté). Des agents sont ainsi affectés à la réalisation de ces différentes tâches qui tiennent essentiellement du régime du distant. On comprend alors que se posent, pour ceux impliqués jusqu'à présent dans le régime du proche, la question de la place de ce mode d'action et celle de la marchandisation de leur intervention, qui, si elle conforte la situation financière de la Chambre, tend aussi à les rabattre sur le régime du distant.

Dans l'activité des conseillers où le régime du distant est dominant, les interactions avec leurs collègues de la Chambre (scène 2) ou les représentants d'autres structures (scène 4) viennent ainsi souvent en premier plan par rapport à celles entretenues avec les agriculteurs (scène 3 ). Les interactions entre pairs permettent, par exemple, de construire des outils de conseil, tels que des guides ou des démarches techniques (usage des phytosanitaires, diagnostic environnemental, élaboration de plan de fumure...), et celles avec les acteurs extérieurs à la Chambre, de produire, par exemple, des références techniques ou de négocier des modes d'intervention auprès des agriculteurs. Des compétences sont déployées dans deux domaines. Dans le domaine technique, il s'agit d'articuler des éléments de connaissances divers pour concevoir des outils de conseil cohérents et utilisables clef en main (par exemple, un outil pour l'élaboration de plans de fumure) ou alors de mobiliser les références de différents services de la Chambre pour réaliser un travail de diagnostic particulier (par exemple, un diagnostic territorial pour une collectivité locale). Dans le domaine social, le but est de construire et de mener des projets en partenariat avec des acteurs multiples, appartenant à des mondes sociaux divers. Les agents doivent savoir conduire des « activités négociées » dans lesquelles les différentes visées et contraintes des partenaires sont prises en compte mais où celles des agriculteurs et de la Chambre orientent aussi la ligne de conduite.

Selon le régime d'action dans lequel sont principalement engagés les conseillers vont alors découler des positionnements différents sur la scène des relations entre agents (scène 2). Sur cette scène ne sont impliqués que des agents de la Chambre dans le cadre de la conduite d'actions en commun, d'échanges de ressources ou de réunions d'équipe, de service ou de direction. Des échanges s'y opèrent, comme dans toute organisation un tant soit peu hiérarchisée, verticalement entre les agents et leur hiérarchie ${ }^{6}$, et horizontalement entre agents. Ces échanges «en interne » leur permettent d'orienter leurs actions, de définir la manière de les conduire et d'accéder aux ressources sociales, cognitives et

6. De manière générale, cette hiérarchie est constituée d'au moins deux niveaux, les responsables de services ou de pôles et la direction, composée d'un directeur épaulé parfois par un directeur adjoint. 
matérielles pour leur mise en œuvre (collègues, acteurs des autres structures, équipements matériels, logiciels, références techniques, données sur les agriculteurs, etc.). Mais ils sont aussi le lieu d'injonctions de la hiérarchie et de positionnements réciproques entre agents. Injonctions de la hiérarchie qui prennent une forme double, quand celles-ci pressent les agents d' « être au service des agriculteurs » tout en «développant la vente de prestations ». Positionnements réciproques entre agents, quand ceux-ci défendent des points de vue différents sur ce que doit être leur métier.

Dans les interactions avec leurs collègues engagés dans le régime du proche, les conseillers principalement impliqués dans le régime du distant se définissent ainsi de deux façons. Lorsqu'ils mènent des actions de service public, ils se présentent comme des porte-parole de l'intérêt général, porte-parole qui incitent les agriculteurs à prendre en compte les nouvelles exigences sociétales et réglementaires, particulièrement dans le domaine environnemental. Quand ils conduisent des actions de service marchand, ils se positionnent comme des défenseurs des intérêts de la Chambre, défenseurs qui instaurent avec les agriculteurs une relation de prestataire de services à client. Une distance d'intérêt avec les agriculteurs apparaît dans leur propos. Inversement, lorsque les agents se positionnent principalement dans leur activité dans le régime du proche, ils se définissent alors comme porte-parole des agriculteurs. En matière environnementale, en particulier, sur des questions de mise en œuvre de nouvelles pratiques ou d'aménagement des exploitations, ils portent et défendent les soucis et difficultés des agriculteurs pour le déploiement de ces changements. Ils peuvent faire ressortir, par exemple, l'impossibilité ou la difficulté pour les agriculteurs de mettre en œuvre des pratiques imposées par la réglementation (cultures intermédiaires, épandage des effluents d'élevage à certaines dates, limitation des traitements phytosanitaires...). Il apparaît alors une sorte de proximité d'intérêt entre ces agents et les agriculteurs. Ces deux positionnements révèlent bien les termes de la double injonction adressée aux agents par leur hiérarchie, " être au service des agriculteurs » et « développer des prestations marchandes ». Les propos des conseillers traduisent, selon leur positionnement dans l'un ou l'autre registre d'action, des formes différentes de mise en tension de ces termes.

\section{Gouvernance et autonomie des Chambres}

Nous avons vu dans le chapitre précédent comment, à partir du positionnement des services des Chambres enquêtées dans les espaces d'activité de notre grille d'analyse, deux profils de Chambre apparaissaient. La différence relevée entre ces profils ne peut manquer de générer des questions sur la manière dont ces organisations peuvent tenir ensemble des services alors que ceux-ci sont animés par des logiques d'action qui divergent. L'analyse de leurs interactions historiques avec d'autres acteurs collectifs tels que les OPA et de la façon dont ces interactions conditionnent leur mode de gouvernance, apporte un certain nombre de réponses.

Si en Saône-et-Loire on observe une concentration des lieux de pouvoir et un élargissement de la diversité des activités propres à la Chambre, en Haute-Savoie, on relève une certaine dispersion de ces lieux en même temps qu'une autolimitation des activités propres. En Saône-etLoire, la Chambre, sous l'impulsion de la Fédération départementale des syndicats d'exploitants agricoles (FDSEA), a été constituée comme une organisation forte aux regards des autres OPA. Elle est un lieu de pouvoir et de débat assez exclusif entre les responsables professionnels élus 
RECHERCHES

de la structure ${ }^{7}$, et un lieu d'affirmation à l'extérieur d'un point de vue relativement unifié. À l'inverse, en Haute-Savoie, c'est l'expression de la diversité des points de vue des différentes OPA qui semble primer. Les responsables professionnels de ces organisations, bien que n'étant pas forcément élus peuvent faire valoir leurs conceptions au sein des commissions interorganismes qui pilotent les services de la Chambre ${ }^{8}$. Les lieux de pouvoir sont beaucoup plus éclatés, la Chambre, en tant que telle, défendant plutôt un point de vue de compromis. Ce statut de quasi-conférence des différentes OPA lui procure une grande plasticité organisationnelle, mais ne lui offre en même temps qu'une faible capacité à mener des actions unifiées et à définir une identité propre.

L'histoire de ces organismes sur ces vingt dernières années fait ainsi apparaître une relative stabilité de la forme de la Chambre de Saône-et-Loire et une relative instabilité de celle de Haute-Savoie - sans qu'il n'y ait ici un quelconque jugement sur l'efficacité de ces formes. Autant la Chambre de Saône-et-Loire s'est développée et s'est

7. Ainsi, au sein de la Chambre de Saône-et-Loire, un Comité de direction, formé des différents chefs de service et du directeur, définit les orientations de travail et la façon de le conduire. Il mène aussi une réflexion plus générale sur l'organisation des activités. Le bureau, constitué d'élus, se réunit très régulièrement pour définir les orientations politiques. Son travail se trouve couplé à celui de la direction par des réunions communes aux deux instances. De plus, des liens plus spécifiques se tissent entre responsables de la Chambre et élus du bureau.

8. En Haute-Savoie, le groupe de direction (le G14) réunit non seulement les responsables de quatre pôles constitutifs de la Chambre mais aussi ceux d'autres organismes. Cette instance tient plus d'un groupe d'information que de décision, les orientations étant définies dans des commissions interorganismes qui pilotent les différents pôles ou services de la Chambre. Y siègent non seulement des élus mais aussi des représentants des autres OPA, des administrations, voire des autres syndicats agricoles. structurée en agrégeant au cours du temps une série de nouvelles missions, autant celle de Haute-Savoie a vécu différents découpages qui l'ont amenée, à proprement parler, à changer de formes ${ }^{9}$ à plusieurs reprises. Une telle situation a bien évidemment des conséquences en termes identitaires. Les agents de la Chambre de Saône-et-Loire, lorsqu'ils sont enquêtés, se reconnaissent ainsi explicitement comme membres d'une même organisation, bien qu'ils n'aient pas tous la même conception du type d'activité à y mettre en œuvre. Si un souci identitaire apparaît, il est plutôt porté par les membres de la direction que par les agents eux-mêmes. Les agents de la Chambre de Haute-Savoie, par contre, mettent en exergue la grande autonomie dont ils disposent dans leur travail et le peu de liens qu'ils entretiennent avec les autres personnels de leur organisme. L'entité Chambre n'est pas un niveau de définition identitaire. Ils font avant tout partie d'un service. Cette autonomie pratique et identitaire peut être complètement assumée par certains agents ou, au contraire, être vécue plus difficilement par d'autres, un manque d'orientation dans le travail à accomplir et un défaut de reconnaissance de celui effectué étant alors mis en relief. Contrairement à ce que l'on observe en Saône-et-Loire, ce sont alors ces agents qui portent un souci constant de l'unité et de l'identité de l'organisation plutôt que les responsables de services ou de pôles, comme si, pour ces derniers, l'éclatement actuel allait de soi.

Le degré de constitution des deux Chambres enquêtées, c'est-à-dire la façon

9. En Haute-Savoie, la Chambre externalise en 1993 dans une association un certain nombre d'activités et réoriente le travail de ses conseillers de secteur vers l'animation de projets de territoire. En 2007, un mouvement contraire à celui d'externalisation est effectué avec la réintégration dans la structure des activités agro-environnementales, cartographie et élevage. En 2009, au moment de notre enquête, la Chambre envisage à nouveau de les externaliser... 
dont elles apparaissent dans leur contexte d'activités comme des structures autonomes, aux contours définis, à l'identité propre, est donc très différent. Si la Chambre de Saône-et-Loire se révèle, en termes organisationnels, être fortement constituée, celle de Haute-Savoie, l'est plutôt faiblement. L'une se caractérise ainsi par son autonomie et l'autre par son hétéronomie, ou autrement dit, par un découplage ou un encastrement par rapport aux autres structures (Grossetti, 2004). À cette autonomie ou hétéronomie correspondrait un regroupement ou un éclatement de leurs différents services dans la grille d'analyse.

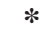

$* *$

$\mathrm{Au}$ terme de cette présentation, nous voudrions souligner le double intérêt de ce travail, l'un de nature théorique et l'autre de nature pratique. Le premier, théorique, repose sur la manière dont, à partir des matériaux empiriques recueillis dans le discours que tiennent les agents sur leur propre activité, nous avons pu faire émerger un cadre d'analyse cohérent qui s'appuie fortement sur celui de la sociologie pragmatique proposé par Laurent Thévenot. Toutefois, n'étant pas bâti à partir des mêmes questionnements, il s'en éloigne aussi, partiellement, en particulier dans la manière de définir un régime du proche centré sur la coordination avec autrui. Ces éléments nous ont permis d'élaborer une grille d'analyse et de faire apparaitre des formes organisationnelles différentes entre les deux Chambres enquêtées. La plus ou moins grande autonomie de ces structures vis-à-vis des autres OPA est apparue un bon critère explicatif des différences enregistrées dans leurs profils. Nous intéressant aux lieux concrets d'interactions entre les acteurs, nous avons, de plus, rendu compte des positionnements réciproques des agents engagés dans l'un et l'autre régime d'action.
Quant à l'intérêt pratique de ce travail, il réside dans l'usage que des agents de Chambres peuvent en faire pour gagner en réflexivité sur leur propre organisation. Il peut en effet leur permettre de s'interroger, à partir du diagnostic réalisé, sur la façon dont leur organisation peut plus ou moins glisser d'un espace d'activités à un autre. Au vu des offres de prestations de conseil payantes des organismes économiques qui émergent, une direction peut ainsi choisir d'investir le domaine d'activité du proche et du marchand. Ce choix vers le développement d'un type d'activités va alors entraîner des conséquences organisationnelles : sectoriser géographiquement les agents. Mais, inversement, un choix organisationnel peut être analysé en fonction de ses conséquences sur les espaces d'activités occupés. La mutualisation en cours, au niveau régional de certaines activités des Chambres départementales, peut être étudiée en ces termes. Placer la plupart des agents à l'échelon régional revient à abandonner les activités qui se situent dans le régime du proche.

L'opérationnalité de l'outil a été testée et validée durant l'année 2012 auprès de l'ensemble des Chambres d'agriculture de la Région Rhône-Alpes. La grille permet aux agents de clarifier leurs activités et les orientations prises par les différents services. Elle fait bien ressortir comment des exigences contradictoires sont, de manière vive, mises en tension. D'une part, il s'agit, pour les Chambres, de conserver une proximité suffisante avec les agriculteurs tout en comptant sur des moyens en diminution et en régionalisant une partie de leur activité et ce, dans un climat de concurrence entre organisations agricoles faisant du conseil. D'autre part, il s'agit pour nombre d'agents de défendre leur mission de développement tout en répondant à une forte injonction à délivrer des prestations payantes ; situation qui induit une hiérarchisation tacite de la valeur du conseil délivré. La grille d'analyse s'est avérée être 
RECHERCHES

un outil suffisamment robuste pour décrire les activités élémentaires de conseil, positionner des services les uns par rapport aux autres et étayer une réflexion plus générale sur une stratégie organisationnelle dans un contexte de reconfiguration du champ du conseil.
Cette recherche a bénéficié du soutien financier de l'Agence Nationale de la Recherche, à travers le projet « Production de connaissances, innovation et développement en agriculture et concrétisation du Développement Durable " (PROD-DD ANR-06-PADD-015).

\section{RÉFÉRENCES BIBLIOGRAPHIQUES}

Albaladejo C., Duvernoy I., Auricoste C. (2009). Quelles formes d'animation territoriale pour les conseillers agricoles ? L'exemple du périurbain albigeois. In Compagnone C., Auricoste C., Lémery B. (Éds), Conseil en agriculture : quelles nouvelles pratiques? Dijon/Paris, Educagri et Éditions Quae, p. 185-200.

Auricoste C., Albaladejo C., Barthe L., Couix N., Duvernoy I., Girard N., Gross H., Labatut J., Lenormand P. (2011). Accompagner l'activité agricole dans les territoires : au carrefour entre le développement sectoriel et le développement territorial. Cahiers Agricultures, $\mathrm{n}^{\circ} 20$, p. 395-399.

Boltanski L., Thévenot L. (1991). De la justification. Les économies de la grandeur. Paris, Gallimard.

Bourdieu P., Wacquant L. (1992). Réponses, Pour une anthropologie réflexive, Paris, Seuil.

Brives H. (2006). Les conseillers agricoles et l'environnement : quelles compétences ? In Rémy J., Brives H., Lémery B. (Éds.), Conseiller en agriculture, Dijon/Paris, Educagri et Éditions Quae, p. 169-182.

Caillé A. (2007). Anthropologie du don. Le tiers paradigme. Paris, La Découverte.

Compagnone C., Golé S. (2011). La reconfiguration de l'espace social du conseil : le cas $\mathrm{du}$ conseil en viticulture bourguignonne. Cahiers Agricultures, ${ }^{\circ} 20$, p. 400-405.

Compagnone C., Lémery B. (2009). Les nouvelles pratiques de développement : une exploration à poursuivre. In Compagnone C., Auricoste C., Lémery B., (Éds.), Conseil en agriculture : quelles nouvelles pratiques?
Dijon/Paris, Educagri et Éditions Quae, p. 235-242.

Compagnone C., Simon B., Moretty P., Kockmann F. (2010). La fin d'un « Yalta du conseil » : le cas du conseil autour de la question des phytosanitaires en Bresse bourguignonne. Colloque SFER Conseiller en agriculture : acteurs, marchés, mutations, 14-15 octobre, Dijon.

Corcuff P. (1998). Justification, stratégie et compassion : apport de la sociologie des régimes d'action, Correspondances (Bulletin d'information scientifique de l'Institut de Recherche sur le Maghreb Contemporain), Tunis, $\mathrm{n}^{\circ} 51$, juin.

De Certeau M. (1990). L'invention du quotidien. Paris, Gallimard.

Faure G., Compagnone C. (2011), Les transformations du conseil face à une nouvelle agriculture. Cahiers Agricultures, $\mathrm{n}^{\circ} 20$, p. 321-326.

Faure G., Desjeux Y., Gasselin P. (2011). Revue bibliographique sur les recherches menées dans le monde sur le conseil en agriculture. Cahiers Agricultures, ${ }^{\circ}$ 20, p. 327-342.

Geertz C. (1999). Savoir local, savoir global. Les lieux du savoir. Paris, Presses universitaires de France.

Genard J.-L. (2011). Investiguer le pluralisme de l'agir. Sociologies [En ligne], Grands résumés, L'Action au pluriel. Sociologie des régimes d'engagement, mis en ligne le 6 juillet 2011, consulté le 13 avril 2012. URL : http://sociologies.revues.org/3574.

Goulet F. (2011). Accompagner et vendre. Les firmes de l'agrofourniture dans l'innovation 
et le conseil en agriculture. Cahiers Agricultures, $\mathrm{n}^{\circ} 20$, p. 382-386.

Grossetti M. (2004). Sociologie de l'imprévisible. Dynamiques de l'activité et des formes sociales. Paris, Presses universitaires de France.

Hellec F. (2009). Des formes méconnues de conseil aux agriculteurs. L'exemple du métier d'inséminateur de bovins. In Compagnone C., Auricoste C., Lémery B. (Éds.) (2009). Conseil en agriculture : quelles nouvelles pratiques? Dijon/Paris, Educagri et Éditions Quae, p. 201-218.

Hughes E. C. (1996). Le regard sociologique. Paris, Éditions de l'EHESS.

Lémery B. (2006). Nouvelle agriculture, nouvelles formes d'exercice et nouveaux enjeux du conseil aux agriculteurs. In Rémy J., Brives H., Lémery B. (Éds.) (2006), Conseiller en agriculture, Dijon/Paris, Educagri et Éditions Quae, p. 235-252.

Mundler P. (2006). Les conseillers d'entreprise entre guichet et projet. In Rémy J., Brives H., Lémery B. (Éds) (2006),
Conseiller en agriculture. Dijon/Paris, Educagri et Éditions Quae, p. 119-136.

Petit S., Compagnone C., Lémery B. Kockmann F., Moretty P. (2011). Les Chambres d'agriculture françaises face à la marchandisation du conseil. Cahiers Agricultures, $\mathrm{n}^{\circ} 20$, p. 321-326.

Rémy J., Brives H., Lémery B. (Éds) (2006). Conseiller en agriculture. Dijon/Paris, Educagri et Éditions Quae.

Ruault C., Lémery B. (2009). Le conseil de groupe dans le développement agricole local : pour quoi faire et comment faire ? In Compagnone C., Auricoste C., Lémery B. (Éds) (2009). Conseil en agriculture : quelles nouvelles pratiques? Dijon/Paris, Educagri et Éditions Quae, p. 71-96.

Strauss A. (1992). La trame de la négociation. Sociologie qualitative et interactionnisme. Traduction d'Isabelle Baszanger, Paris, L'Harmattan.

Thévenot L. (2006). L'action au pluriel. Sociologie des régimes d'engagement. Paris, La Découverte. 